\title{
Major Periods and Cultural Epochs of Japanese History
}

Jōmon period

Yayoi period

Tomb period

Age of Reform

Asuka epoch (552-645)

Hakuhō epoch (645-710)

Nara period

Tempyō epoch (mid-eighth century)

Heian period

Jōgan epoch (mid- to late ninth century)

Fujiwara epoch (tenth century to late eleventh century)

Kamakura period

Kemmu Restoration

Kitayama epoch (late fourteenth and early fifteenth centuries)

Higashiyama epoch (second half of the fifteenth century)

Age of Unification

Azuchi-Momoyama epoch (1568-1600 or 1615)

Namban epoch (late sixteenth and early seventeenth centuries)

Tokugawa (Edo) period

$1600-1867$

Genroku epoch (ca. 1675-1725)

Bunka-Bunsei epoch (late eighteenth and early nineteenth centuries)

Meiji period

1868-1911

$1912-1925$

1926-

Shōwa period
1333-1336

1336-1573

ca. $8000-300$ B.C.

ca. 300-552

552-710

$710-784$

$794-1185$

$1568-1600$ 